\title{
WILEY-VCH
}

DOI: 10.1002/((please add manuscript number))

Article type: Communication

\section{Endocytic Capsule Sensors for Probing Cellular Internalization}

Kang Liang, Sylvia T. Gunawan, Joseph J. Richardson, Georgina K. Such, Jiwei Cui, and Frank Caruso*

K. Liang, S. T. Gunawan, J. J. Richardson, Dr. J. Cui, and Prof F. Caruso

Department of Chemical and Biomolecular Engineering, The University of Melbourne, Parkville, Victoria 3010, Australia

E-mail: fcaruso@unimelb.edu.au

Dr. G. K. Such

School of Chemistry, The University of Melbourne, Parkville, Victoria 3010, Australia

Keywords: ((internalization sensors, polymer capsules, layer-by-layer, cell uptake, nanotechnology))

The targeted delivery of therapeutic molecules into cells is important for improving treatments for a range of diseases. ${ }^{1}$ Polymer carriers can serve as suitable systems for protecting and delivering fragile or toxic therapeutics. ${ }^{2}$ To achieve next-generation treatments based on these technologies, it is important that fundamental carrier properties such as carriercell interactions and internalization mechanisms are elucidated. ${ }^{3}$ However, current technologies for probing carrier internalization are limited by either low throughput or low sensitivity, primarily due to the nature of the fluorescent probes. ${ }^{4}$

In most mammalian cells, micron-sized polymer carriers are typically internalized through endocytic pathways, ${ }^{5}$ where the $\mathrm{pH}$ changes from neutral outside the cells ( $\left.\mathrm{pH} 7.4\right)$ to acidic in the endosomes and lysosomes (pH 4.7-6.3). ${ }^{6} \mathrm{pH}$-sensitive fluorescent probes, such as pHrodo $^{\mathrm{TM}}$, SNARF ${ }^{\circledR}$, or some fluorescence resonance energy transfer (FRET) pairs can be conjugated to the carriers to detect internalization-coupled $\mathrm{pH}$ changes using flow cytometry, ${ }^{7}$ where internalization is indicated by a shift in fluorescence signals or intensities. Several capsule-based sensors utilizing these fluorescence molecules have been reported in the past. ${ }^{8}$ However, these probes often require extensive calibration and also create difficulties in 


\section{WILEY-VCH}

distinguishing between a large number of carriers attached to the cell surface and a small number of internalized carriers (and vice versa). Common internalization assays that use trypan blue or acid wash, to quench or remove surface-bound materials, are often at the expense of specificity and may alter the association signal in the latter method. ${ }^{4}$ Additionally, imaging techniques, such as high-resolution microscopy and imaging flow cytometry, can be used to determine internalization. However, there is a trade off between resolution and throughput, as the advantages have not yet been synergistically combined. Therefore, it is of interest to design a modular, high-throughput system that does not require additional treatment, for unambiguously determining the internalization of carriers in a complex biological environment.

Herein, we report the synthesis of polymer capsules with an in-built pH-based fluorescent sensor that allows a highly sensitive and high-throughput study of carrier internalization in living cells. To utilize the $\mathrm{pH}$ change associated with the internalization of the polymer carriers, poly (2-(diisopropylamino)ethyl methacrylate) (PDPA) (pK $\left.K_{\mathrm{a}} \sim 6.4\right)$ was chosen as the building-block. Recently, several studies have employed PDPA for the design of novel responsive polymer carriers, as PDPA undergoes a transition from hydrophobic to hydrophilic, which occurs in the $\mathrm{pH}$ range of cellular internalization. ${ }^{9}$ We previously reported the layer-bylayer (LbL) assembly of pH-responsive PDPA multilayer capsules that are capable of swelling upon internalization in intracellular acidic compartments. ${ }^{10}$ Herein, we exploit the size-responsiveness of PDPA capsules to design capsules that contain a fluorescence probe that switches 'on' and 'off' with $\mathrm{pH}$. This fluorescence sensor has important application for unambiguously probing the high-throughput internalization of capsules (Scheme 1). Utilizing this system, we determine, for the first time, the real time size-dependent binding versus internalization kinetics of polymer capsules. 


\section{WILEY-VCH}

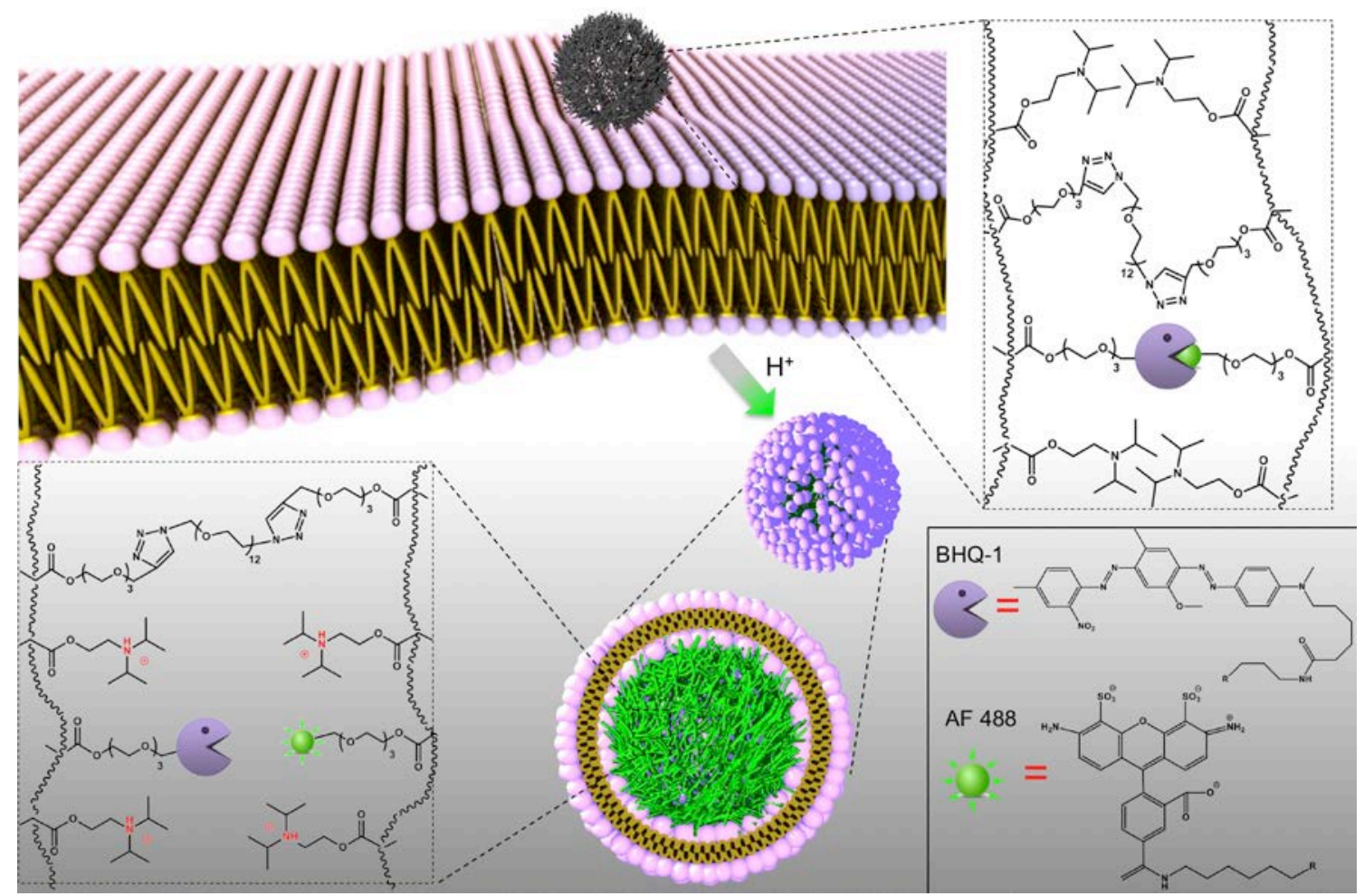

Scheme 1. Capsules with in-built fluorescent on/off switches. At neutral pH (e.g., extracellular), the multilayer capsules shrink, causing strong fluorescence quenching (black); at acidic $\mathrm{pH}$ (e.g., intracellular endosomal or lysosomal conditions), the capsules swell, leading to fluorescence recovery (green).

Alexa Fluor 488 (AF488) azide (fluorophore) or Black Hole Quencher 1 (BHQ1) azide (quencher) were conjugated to alkyne-containing PDPA (PDPA Alk) via copper-catalyzed azide-alkyne cycloaddition (CuAAC), followed by dialysis and freeze-drying to yield dye- or quencher-conjugated polymers, $\mathrm{PDPA}_{\mathrm{AF} 488}$ and PDPA $\mathrm{B}_{\mathrm{BHQ} 1}$, respectively. The dye or quencher was mixed with PDPA $_{\text {Alk }}$ at a 1:200 ratio, allowing the majority of alkynes to remain unreacted and available for the subsequent crosslinking of multilayer films. Success of the dye or quencher coupling was indicated by attenuated total reflectance Fourier transform infrared (ATR FT-IR) spectroscopy through the appearance of absorbance bands in the range of $1585-2000 \mathrm{~cm}^{-1}$, which are characteristic of the aromatic systems of the dye and quencher (data not shown). The sensing capsules were synthesized via LbL assembly between poly(methacrylic acid) (PMA) and alternate layers of PDPA $\mathrm{BHQ}_{1}$ and PDPA $\mathrm{AF}_{888}$ on silica particles $\left(2.6 \mu \mathrm{m}\right.$ in diameter) at $\mathrm{pH} 4$ (i.e., $\mathrm{PDPA}_{\mathrm{BHQ} 1} / \mathrm{PMA} / \mathrm{PDPA}_{\mathrm{AF} 488} / \mathrm{PMA}$ alternate 


\section{WILEY-VCH}

multilayers). We have previously reported the homogeneous layer buildup of this polymer pair on both planar and particle surfaces. ${ }^{10 \mathrm{~b}}$ After consecutive deposition of five bilayers, the multilayers were cross-linked via CuAAC between the free alkyne moieties in the PDPA Alk layers and a bisazide cross-linker. After the removal of the silica template by hydrofluoric acid and removal of the PMA layers by increasing the $\mathrm{pH}$ to 7.4 , hollow PDPA capsules with alternate fluorescence/quencher layers were formed. Our previous work showed PDPA capsules have a zeta-potential close to $0 \mathrm{mV}$ at $\mathrm{pH}$ 7.4, and that they become positively charged $(\sim 25 \mathrm{mV})$ at intracellular $\mathrm{pH}(<6.4)$ due to the hydrophobic-to-hydrophilic transition of PDPA at its $\mathrm{p} K_{\mathrm{a}}(\sim 6.4) .{ }^{10 \mathrm{~b}}$

To demonstrate the in-built pH-coupled fluorescence switch, the capsules were incubated in pH-adjusted phosphate-buffered saline (PBS) buffers and examined using fluorescence microscopy. At least 50 capsules were sized at each $\mathrm{pH}$ using a fluorescence microscope, and the PDI ( 0.04 at both pH 7.4 and 6.0) was calculated accordingly. At extracellular pH (7.4), the capsules shrunk when compared to the size of the silica templates (from 2.6 to $1.6 \mu \mathrm{m}$ ), and negligible fluorescence was observed (Figure 1, right insets). This shrinkage is probably due to the hydrophobic interactions between PDPA multilayers, which cause the quencher and fluorophore in the adjacent PDPA layers to be in close proximity, leading to fluorescence quenching. In contrast, at the $\mathrm{pH}$ of early endosomes ( $\sim 6.0)$, the capsules instantly $(<1 \mathrm{~s})$ swelled to $\sim 3 \mu \mathrm{m}$ and a bright fluorescence signal was observed (Figure 1, left insets). This swelling is likely due to the charge repulsion between protonated PDPA layers, which allow the fluorophore and quenching molecules to separate, thereby giving higher fluorescence signals (Figure 1, left insets). The pH-induced fluorescence 'on/off' switch was also assessed across a wide $\mathrm{pH}$ range (from 4.0 to 9.0) using flow cytometry. A sharp increase in the fluorescence intensity and capsule diameter was observed by moving from $\mathrm{pH} 6.5$ to 6.2 (Figure 1, Supporting Information Figure S1), suggesting that the PDPA shifted from hydrophobic to hydrophilic around its $\mathrm{pK}_{\mathrm{a}} \cdot{ }^{10 \mathrm{~b}}$ Furthermore, the capsules retained their $\mathrm{pH}$ - 


\section{WILEY-VCH}

induced fluorescence 'on/off' switching even after three consecutive alternating $\mathrm{pH}$ washes in pH-adjusted PBS (Supporting Information Figure S2).

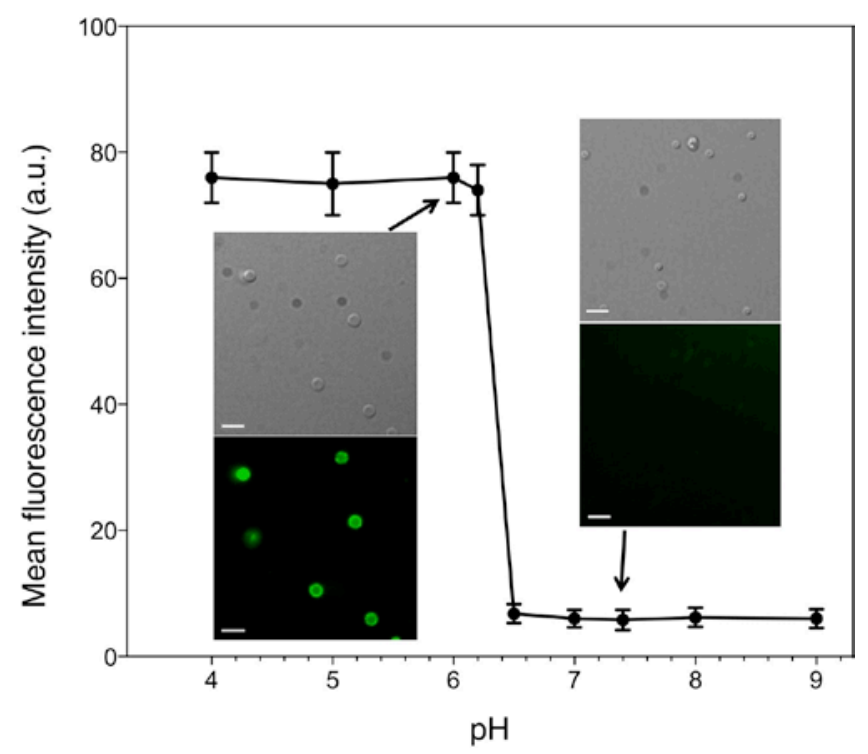

Figure 1. Fluorescence intensity of the capsules at different $\mathrm{pH}$ values, assessed by flow cytometry. Measurements were performed in triplicate. Bright field (differential interference contrast, DIC) and fluorescence microscopy images of sensing capsules dispersed in $\mathrm{pH} 6.0$ (left insets) and $\mathrm{pH} 7.4$ PBS (right insets). Scale bars $=5 \mu \mathrm{m}$.

To demonstrate the feasibility of the capsules as internalization sensors, they were incubated with JAWS II cells, an immortalized immature dendritic cell (DC) line harvested from mouse bone marrow. DCs play a critical role in activating both $\mathrm{T}$ and $\mathrm{B}$ cells during an immune response, ${ }^{11}$ and have been widely studied as a target cell line for immunotherapy against cancers and infectious diseases. ${ }^{12}$ Internalization was initially assessed using deconvolution optical microscopy (Figure 2), which provides z-axis resolution up to 150 nm. ${ }^{13}$ Alexa Fluor 647, which is not quenchable by BHQ1, was post-conjugated to the capsules after the formation of sensing capsules to enable the constant monitoring of the capsules, irrespective of $\mathrm{pH}$ for cell association and subsequent internalization. After $2 \mathrm{~h}$ incubation, capsules inside the cell membrane swelled and emitted a strong AF488 signal (Figure 2a). As expected, the AF647 signal was observed from all capsules, irrespective of their location outside or inside the cell membrane (Figure 2b). However, capsules located outside of the cellular membrane remained shrunk and did not emit AF488 fluorescence 


\section{WILEY-VCH}

(Figure 2a). Further evidence that the capsules were internalized in acidic cellular compartments was gained after subsequent incubation of the cells containing the capsules for

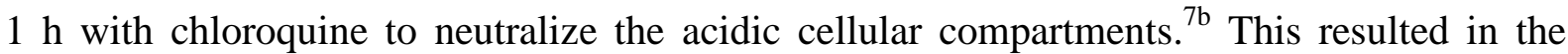
capsules shrinking and losing their green fluorescence (Supporting Information, Figure S3).
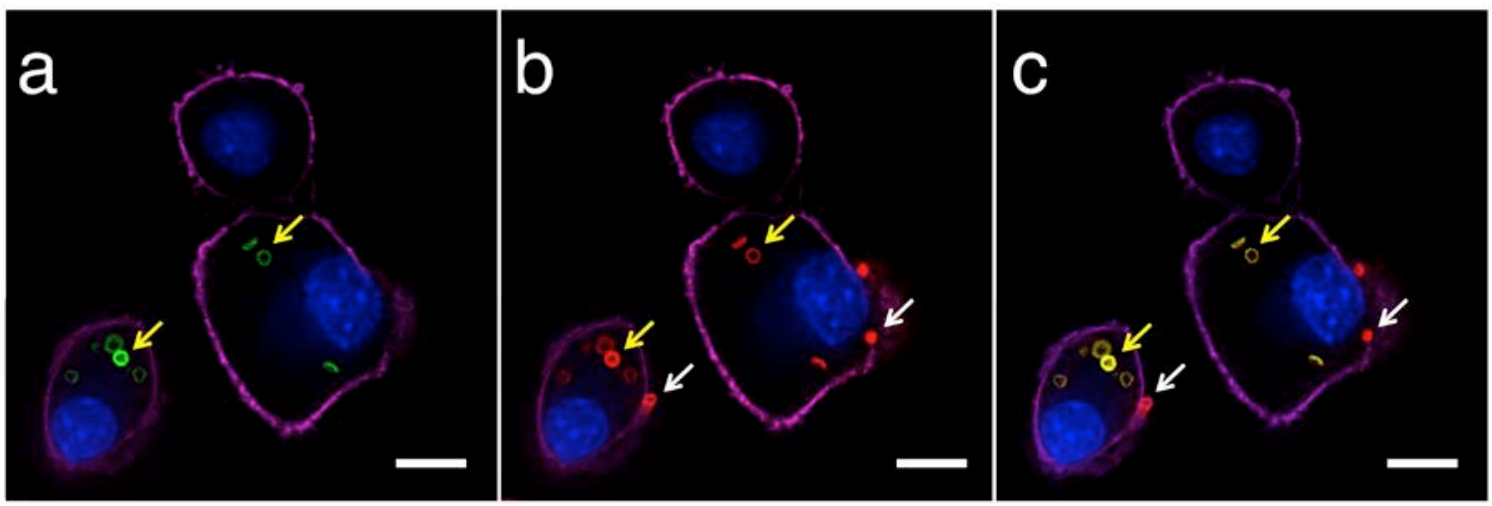

Figure 2. Deconvolution microscopy images of JAWS II cells incubated with sensing capsules for 2 h. a) Capsules showing Alexa Fluor 488 fluorescence, b) capsules showing Alexa Fluor 647 fluorescence, c) overlay. White arrows indicate capsules associated with the cells but not internalized. Yellow arrows indicate the internalized capsules. Alexa Fluor 488 green, Alexa Fluor 647 red, DAPI blue, and wheat germ agglutinin purple. Scale bars $=10$ $\mu \mathrm{m}$.

Flow cytometry was employed to demonstrate that the sensing capsules are applicable for high-sensitivity and high-throughput internalization studies. Capsules prepared from silica particles with diameters of $1.1 \mu \mathrm{m}, 2.6 \mu \mathrm{m}$, and $5 \mu \mathrm{m}$, had diameters of $600 \mathrm{~nm}, 1.6 \mu \mathrm{m}$, and 3 $\mu \mathrm{m}$ in PBS (pH 7.4), respectively (Supporting Information, Figures S4 and S5). The capsuleto-cell ratio for incubation was chosen as $20: 1$, and to be the same across the different-sized capsules. After 2, 4, 6, 18, and 24 h incubation, the cells were washed to remove unbound capsules and were assessed using flow cytometry. The BHQ-1 insensitive dye AF647 was used to monitor the association kinetics of membrane-binding and internalization of the capsules (i.e., the total number of capsules that interacted with cells). Capsule association kinetics demonstrated size-dependent behavior: smaller capsules were observed to associate more with the JAWS II cells over time (Figure 3, Supporting Information Figure S6). This is probably due to the overall larger surface area in contact with the cell membrane for the smaller capsules. ${ }^{14}$ Furthermore, the capsules also demonstrated size-dependent 


\section{WILEY-VCH}

internalization kinetics. The fluorescence probe demonstrated that the rate of cells (with AF488) signals increased more rapidly for smaller capsules (Figure 3). For $600 \mathrm{~nm}$ capsules, binding to the cell membrane led to effective internalization (i.e., $80 \%$ of the cells showed internalized capsules at $24 \mathrm{~h}$ ). However, although the cellular association of larger capsules (1.6 and $3 \mu \mathrm{m}$ ) showed a similar percentage of above $80 \%$ compared with the $600 \mathrm{~nm}$ capsules at $24 \mathrm{~h}$, only $\sim 40-50 \%$ of the cells showed internalization of larger capsules. This result demonstrates that the cellular internalization rate of polymer carriers is size-dependent, which is in agreement with previous studies. ${ }^{14-15}$ This is probably in part due to the higher energy requirements involved in the active internalization process of larger materials and/or different routes of endocytosis. ${ }^{14,16}$ It is noted that the real-time monitoring of the capsule internalization was probably slightly underestimated due to the inherent lag in the endosomal acidification process, which usually occurs within a few minutes of the formation of new endosomes. ${ }^{17}$ Polymers with a higher $\mathrm{p} K_{\mathrm{a}}$ could be used to further increase the sensitivity during this lag time for improved resolution of the internalization kinetics.

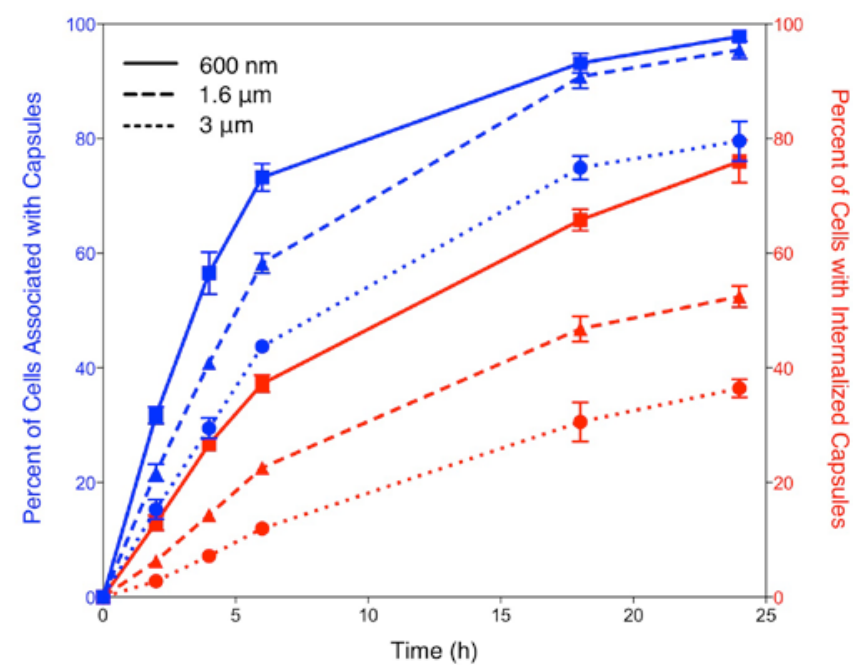

Figure 3. Kinetics of association (blue, left axis) and internalization (red, right axis) of sensing capsules within JAWS II cells. Measurements were performed in triplicate.

In conclusion, we have synthesized a new class of polymer capsules with in-built pH-coupled fluorescence switches, which are particularly useful for monitoring cellular internalization. The reported system provides a highly sensitive approach for studying cell interactions with 


\section{WILEY-VCH}

high-throughput, using standard instrumentation without complicated calibration procedures.

Using flow cytometry, we demonstrated the high-throughput quantification between surfacebound and internalized capsules, which provides fundamental information for understanding the interactions between particles and biological systems at a cellular level.

\section{Experimental Section}

Details of material and methods, including polymer synthesis, capsule preparation and characterization and cell experiments are described in the Supporting Information.

\section{Supporting Information}

Supporting Information is available from the Wiley Online Library or from the author.

\section{Acknowledgements}

This work was supported by the Australian Research Council under the Australian Laureate Fellowship (F.C., 120100030), Discovery Project (F.C., 130101846), Super Science (F.C., FS110200025), and Future Fellowship (G.K.S., FT120100564) schemes.

Received: ((will be filled in by the editorial staff))

Revised: ((will be filled in by the editorial staff)) Published online: ((will be filled in by the editorial staff))

[1] a) Canton, I.; Battaglia, G., Chem. Soc. Rev. 2012, 41, 2718; b) Marsh, M.; Helenius, A., Cell 2006, 124, 729.

[2] a) De Koker, S.; Hoogenboom, R.; De Geest, B. G., Chem. Soc. Rev. 2012, 41, 2867;

b) Devadasu, V. R.; Bhardwaj, V.; Kumar, M. N. V. R., Chem. Rev. 2012, 113, 1686; c) De Koker, S.; De Cock, L. J.; Rivera-Gil, P.; Parak, W. J.; Velty, R. A.; Vervaet, C.; Remon, J. P.; Grooten, J.; De Geest, B. G., Adv. Drug Delivery Rev. 2011, 63, 748; d) del Mercato, L. L.; Rivera-Gil, P.; Abbasi, A. Z.; Ochs, M.; Ganas, C.; Zins, I.; Sonnichsen, C.; Parak, W. J., Nanoscale 2010, 2, 458; e) Hammond, P. T., Nanomedicine 2012, 7, 619; f) Such, G. K.; Johnston, A. P. R.; Caruso, F., Chem. Soc. Rev. 2011, 40, 19. 


\section{WILEY-VCH}

[3] Yan, Y.; Björnmalm, M.; Caruso, F., Chem. Mater. 2014, 26, 452.

[4] Liu, H.; Johnston, A. P. R., Angew. Chem., Int. Ed. 2013, 52, 5744.

[5] a) Duncan, R., Nat. Rev. Cancer 2006, 6, 688; b) Hillaireau, H.; Couvreur, P., Cell.

Mol. Life Sci. 2009, 66, 2873; c) Muñoz Javier, A.; Kreft, O.; Semmling, M.; Kempter, S.;

Skirtach, A. G.; Bruns, O. T.; del Pino, P.; Bedard, M. F.; Rädler, J.; Käs, J.; Plank, C.;

Sukhorukov, G. B.; Parak, W. J., Adv. Mater. 2008, 20, 4281; d) Yan, Y.; Ochs, C. J.; Such,

G. K.; Heath, J. K.; Nice, E. C.; Caruso, F., Adv. Mater. 2010, 22, 5398; e) Rivera-Gil, P.; De

Koker, S.; De Geest, B. G.; Parak, W. J., Nano Lett. 2009, 9, 4398.

[6] a) Casey, J. R.; Grinstein, S.; Orlowski, J., Nat. Rev. Mol. Cell Biol. 2010, 11, 50; b)

Maxfield, F. R.; McGraw, T. E., Nat. Rev. Mol. Cell Biol. 2004, 5, 121.

[7] a) Li, Z.; Wu, S.; Han, J.; Han, S., Analyst 2011, 136, 3698; b) Kreft, O.; Javier, A.

M.; Sukhorukov, G. B.; Parak, W. J., J. Mater. Chem. 2007, 17, 4471; c) Han, J.; Burgess, K., Chem. Rev. 2009, 110, 2709.

[8] a) Reibetanz, U.; Haložan, D.; Brumen, M.; Donath, E., Biomacromolecules 2007, 8, 1927; b) De Koker, S.; De Geest, B. G.; Singh, S. K.; De Rycke, R.; Naessens, T.; Van Kooyk, Y.; Demeester, J.; De Smedt, S. C.; Grooten, J., Angew. Chem., Int. Ed. 2009, 48, 8485; c) del Pino, P.; Munoz-Javier, A.; Vlaskou, D.; Rivera Gil, P.; Plank, C.; Parak, W. J., Nano Lett. 2010, 10, 3914.

[9] a) Du, J.; Armes, S. P., J. Am. Chem. Soc. 2005, 127, 12800; b) Lomas, H.; Canton, I.; MacNeil, S.; Du, J.; Armes, S. P.; Ryan, A. J.; Lewis, A. L.; Battaglia, G., Adv. Mater. 2007, 19, 4238; c) Giacomelli, F. C.; Stepanek, P.; Giacomelli, C.; Schmidt, V.; Jager, E.; Jager, A.; Ulbrich, K., Soft Matter 2011, 7, 9316; d) Peng, C.-L.; Yang, L.-Y.; Luo, T.-Y.; Lai, P.-S.; Yang, S.-J.; Lin, W.-J.; Shieh, M.-J., Nanotechnology 2010, 21, 155103.

[10] a) Liang, K.; Such, G. K.; Zhu, Z.; Dodds, S. J.; Johnston, A. P. R.; Cui, J.; Ejima, H.; Caruso, F., ACS Nano 2012, 6, 10186; b) Liang, K.; Such, G. K.; Zhu, Z.; Yan, Y.; Lomas, H.; Caruso, F., Adv. Mater. 2011, 23, H273. 


\section{WILEY-VCH}

[11] Steinman, R. M., Annu. Rev. Immunol. 1991, 9, 271.

[12] Jiang, X.; Shen, C.; Rey-Ladino, J.; Yu, H.; Brunham, R. C., Infect. Immun. 2008, 76, 2392.

[13] Sibarita, J.-B., Deconvolution Microscopy. In Microscopy Techniques, Rietdorf, J., Ed. Springer Berlin Heidelberg: 2005; Vol. 95, pp 201-243.

[14] Gratton, S. E. A.; Ropp, P. A.; Pohlhaus, P. D.; Luft, J. C.; Madden, V. J.; Napier, M. E.; DeSimone, J. M., Proc. Natl. Acad. Sci. U. S. A. 2008, 105, 11613.

[15] Kumari, S.; Mg, S.; Mayor, S., Cell Res. 2010, 20, 256.

[16] Schmid, S. L.; Carter, L. L., J. Cell Biol. 1990, 111, 2307.

[17] a) Huotari, J.; Helenius, A., EMBO J. 2011, 30, 3481; b) Blanchette, C. D.; Woo, Y.H.; Thomas, C.; Shen, N.; Sulchek, T. A.; Hiddessen, A. L., PLoS ONE 2009, 4, e6056; c) Cain, C. C.; Sipe, D. M.; Murphy, R. F., Proc. Natl. Acad. Sci. U. S. A. 1989, 86, 544. 


\section{WILEY-VCH}

A new class of polymer capsules with in-built endocytic $\mathrm{pH}$-coupled fluorescence switches is reported. These capsules display reversible 'on/off' fluorescence in response to cellular $\mathrm{pH}$ variations. Using this system, we demonstrated the high-throughput quantification between surface-bound capsules and internalized capsules. This system allows the fundamental study of the interaction between nanoengineered materials and biological systems at a cellular level.

Keywords: internalization sensors, polymer capsules, layer-by-layer, cell uptake, nanotechnology

K. Liang, S. T. Gunawan, J. J. Richardson, G. K. Such, J. Cui, F. Caruso*

\section{Endocytic Capsule Sensors for Probing Cellular Internalization}

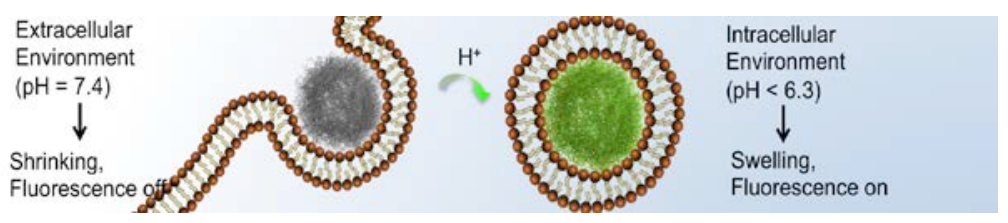




\section{University Library}

\section{- M M N E R VA A gateway to Melbourne's research publications}

Minerva Access is the Institutional Repository of The University of Melbourne

Author/s:

Liang, K;Gunawan, ST;Richardson, JJ;Such, GK;Cui, J;Caruso, F

Title:

Endocytic Capsule Sensors for Probing Cellular Internalization

Date:

2014-10-01

Citation:

Liang, K., Gunawan, S. T., Richardson, J. J., Such, G. K., Cui, J. \& Caruso, F. (2014).

Endocytic Capsule Sensors for Probing Cellular Internalization. ADVANCED HEALTHCARE MATERIALS, 3 (10), pp.1551-1554. https://doi.org/10.1002/adhm.201400139.

Persistent Link:

http://hdl.handle.net/11343/123267 